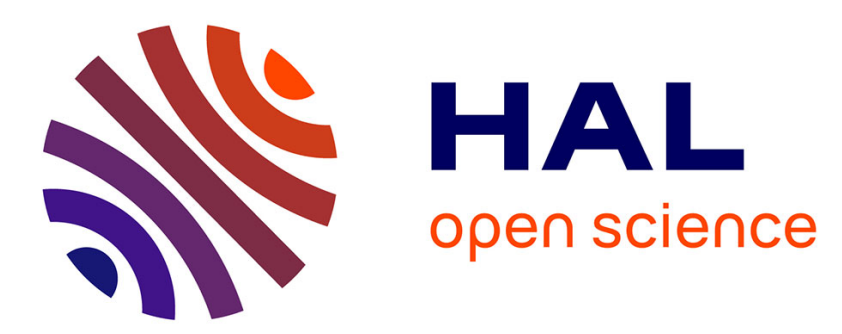

\title{
A Near-Optimal Multiuser Detector for MC-CDMA systems Using Geometrical Approach
}

Amor Nafkha, Christian Roland, Emmanuel Boutillon

\section{To cite this version:}

Amor Nafkha, Christian Roland, Emmanuel Boutillon. A Near-Optimal Multiuser Detector for MCCDMA systems Using Geometrical Approach. ICASSP, 2005, Philadelphia, United States. pp.877880. hal-00068921

\section{HAL Id: hal-00068921 https://hal.science/hal-00068921}

Submitted on 15 May 2006

HAL is a multi-disciplinary open access archive for the deposit and dissemination of scientific research documents, whether they are published or not. The documents may come from teaching and research institutions in France or abroad, or from public or private research centers.
L'archive ouverte pluridisciplinaire HAL, est destinée au dépôt et à la diffusion de documents scientifiques de niveau recherche, publiés ou non, émanant des établissements d'enseignement et de recherche français ou étrangers, des laboratoires publics ou privés. 


\title{
A NEAR-OPTIMAL MULTIUSER DETECTOR FOR MC-CDMA SYSTEMS USING GEOMETRICAL APPROACH
}

\author{
A. Nafkha, C. Roland, E. Boutillon \\ LESTER, CNRS FRE 2734 - UBS University BP 92116 - 56321 Lorient France \\ E-mail: \{amor.nafkha, christian.roland, emmanuel.boutillon\}@ univ-ubs.fr
}

\begin{abstract}
An efficient sub-optimal algorithm, called HIS (Hyperplane Intersection and Selection) detection algorithm, is proposed to solve the problem of joint detection of $K$ users in a MCCDMA system. Compared to the existing solutions, the proposed algorithm has three characteristics very attractive for pratical systems. Firstly, it has nearly optimal performance. Secondly, it has a low computational complexity $\left(\mathcal{O}\left(K^{2}\right)\right.$ multiplications and $\mathcal{O}\left(K^{3}\right)$ additions). Third, the algorithm has an inherent parallelism. To our knowledge, the HIS algorithm is not just an add-on to a previous existing algorithm but a rather new decoding technic based on a singular value decomposition of the channel matrix $H$. After giving the equation of the MC-CDMA multi-user detection problem, the HIS algorithm is described. Its performance is compared to known existing algorithms (ZF, MMSE, PIC and Sphere Decoding). For a BER as low as $10^{-4}$, the HIS algorithm introduces only $0.2 d B$ degradation compared to the optimal Sphere Decoding algorithm for $K=16$ users againt $3.8 d B$ for the PIC (with two MMSE stages) algorithm.
\end{abstract}

\section{INTRODUCTION}

Multi-Carrier Code Division Multiple Access (MC-CDMA) was first proposed in [1] as a multiple access modulation technique which combines the advantage of the Orthogonal Frequency Division Multiplexing (OFDM) and the Direct Sequence Code Division Multiple Access (DS-CDMA) analysed and developed in [2] and [3]. Although MC-CDMA is an attractive candidate for future wireless communication, a lot of challenges should be solved before it can achieve its full potential. One of these challenges is the multiuser detection design.

To overcome the multiuser interference introduced by the transmission on a multipath channel, various single user and multiuser detection techniques have been proposed [4]. Among them, the optimum multiuser detection, based on a Maximum Likelihood (ML) exhaustive search [5], has a prohibitive complexity, which grow exponentially with the number of users. The techniques for multi-user detection in
MC-CDMA can be classified as linear or non-linear techniques. A number of these techniques have evolved from previous research for CDMA-based systems. A new suboptimal detection algorithm having low complexity for MCCDMA systems is proposed in the present paper. This algorithm, called the Hyperplane Intersection and Selection algorithm, has an important parallelism level which allows us to predict an efficient hardware implementation. The proposed algorithm can yield nearly a ML performance with low computational complexity.

The paper is organised as follows: Section 2 describes the synchronous MC-CDMA system and the corresponding lattice representation. In section 3, a brief summary of the various linear and non-linear multi-user detection techniques are presented. The HIS algorithm is explained in section 4. Complexity and simulation results for a MC-CDMA system are presented in section 5 and compared to the performance of some sub-optimum detection algorithms. Finally, some conclusions are drawn in section 6.

\section{THE SYNCHRONOUS MC-CDMA SYSTEM MODEL}

Let us consider a synchronous MC-CDMA system with $K$ users as described in [6]. At time $t$, the transmitted symbol $x_{k}(t)$ of the user $k$ is spread by a signature $\mathbf{c}_{k}=\left(c_{k 1}, . ., c_{k L}\right)$, which has good cross-correlation properties with other signatures. In this paper, signatures belong to an orthogonal Walsh-Hadamard set of size $L$. The $L$ obtained chips are transmitted on the $L$ different sub-carriers of an OFDM modulation symbol. Let $s_{k}(t)$ denote the modulated signal filtered by a frequency selective multipath channel. After addition of interfering user signals $\sum_{p \neq k} s_{p}(t)$ and Additive White Gaussian Noise, an OFDM demodulation is performed. The channel is assumed non frequency selective on the sub-carrier bandwidth.

In downlink, all users share the same channel defined by $\mathcal{H}(t)=\operatorname{diag}\left(h_{1}(t), . ., h_{L}(t)\right)$. At each time $t$, the coefficients $h_{q}(t)$ where $q \in\{1 . . L\}$ are modeled by independent Gaussian random variables of variance 0.5 per dimension. The $L \times K$ matrix combining the spreading and the channel 
effects for all users can be expressed as:

$$
C(t)=\left(\begin{array}{ccc}
c_{11} h_{1}(t) & \ldots & c_{K 1} h_{1}(t) \\
\vdots & \vdots & \vdots \\
c_{L L} h_{L}(t) & \ldots & c_{K L} h_{L}(t)
\end{array}\right)
$$

At time $t$, the received vector $R(t)=\left(r_{1}(t), . ., r_{L}(t)\right)^{T}$ may be written as:

$$
R(t)=C(t) \times D(t)+B(t)
$$

where vector $D(t)=\left(d_{1}(t), . ., d_{K}(t)\right)^{T}$ contains the $K$ transmitted symbols (4-QAM constellation will be used) and $B(t)=\left(b_{1}(t), \ldots, b_{L}(t)\right)^{T}$ is the AWGN vector with variance $\sigma^{2}$ per dimension. We assume perfect knowledge of the channel state information (CSI) by the receiver. Equation (1) can be expressed in the real domain as:

$$
Y(t)=H(t) \times X(t)+\nu(t)
$$

where:

$$
\begin{aligned}
H(t) & =\left[\begin{array}{cc}
r e(C(t)) & -i m(C(t)) \\
i m(C(t)) & \operatorname{re}(C(t))
\end{array}\right] \\
X(t) & =\left[\begin{array}{ll}
r e(D(t)) & i m(D(t))
\end{array}\right]^{T} \\
Y(t) & =\left[\begin{array}{ll}
r e(R(t)) & i m(R(t))
\end{array}\right]^{T} \\
\nu(t) & =\left[\begin{array}{ll}
r e(B(t)) & i m(B(t))
\end{array}\right]^{T}
\end{aligned}
$$

$r e(V)$ and $i m(V)$ denotes respectively the real and the imaginary part of the vector $V$. Note that the rank of the matrix $H$ is generally $2 \times \min (L, K)$ so the colums of $H$ formed a basis vectors of a lattice lying in a $2 K$-dimensional space.

The optimal solution of (2) for minimizing the Bit Error Rate (BER) is given by the ML decoder [2] which solves the integer least squares (ILS) problem:

$$
\hat{X}(t)_{m l}=\arg \min _{X \in \xi}\|Y(t)-H(t) \cdot X\|_{2}^{2}
$$

where $N=2 K$ and $\xi=\{-1,1\}^{N}$. The optimal block symbol decoding via exhaustive search in (3) implies a complexity of $O\left(2^{N}\right)$ which is exponential in $N$.

\section{REVIEW OF DETECTION ALGORITHMS}

In the following, sub-optimal linear and nonlinear detection schemes with reduced complexity are presented.

\subsection{Linear detection}

Linear multiuser detectors have been extensively studied during the past decade [7]. Here, a linear transformation is applied to the received signal in order to remove or at least to reduce Multi-User Interferences (MUI). Recalling the output of the matched filter $X_{Z F}=\left(H^{t} H\right)^{-1} H^{t} Y=$ $X+\left(H^{t} H\right)^{-1} H^{t} \nu$. This receiver is called zero forcing detector or decorrelator. A better strategy to suppress the MUI is to minimize the Mean Squared Error (MSE) between the actually transmitted symbols $X$ and the estimates $X_{M M S E}$ The solution to this problem is known as a MMSE detector and differs from the decorrelator only by adding the noise power to the main diagonal of the matrix to be inversed $X_{M M S E}=\left(H^{t} H+\sigma^{2} I_{N}\right)^{-1} H^{t} Y$.

\subsection{Nonlinear detection}

To improve the performance of the multiuser detection, non linearity techniques should be introduced. Nonlinear suboptimal multiuser receivers can be divided into three categories: decision feedback, multistage and other nonlinear receivers. Successive Interference Cancellation receivers [8], which are a subclass of decision feedback receivers, can also be called "re-generative" IC algorithms. It estimates and regenerates the signal waveform of an interfering user at chip level and subtract it from the received baseband signal to enhance detection of other users. In the case of Parallel Interference Cancellation PIC [9], which belongs to multistage class, the transmitted signal of each user is detected in parallel over a number of stages. There are several other nonlinear techniques, which are less popular like Semi-Define Programming SDP described in [10].

\section{DESCRIPTION OF THE "HIS" ALGORITHM}

In this section, the proposed multiuser detection algorithm is described.

\subsection{Basic idea}

In what follows, the singular value decomposition (SVD) of channel matrix $H=U \Sigma V^{*}$ is used, where the diagonal matrix $\Sigma$ contains the singular values $\left(\left\{\lambda_{p}\right\}_{p=1 . . N}\right)$ and the matrices $U$ and $V$ contain, respectively, the left $\left\{u_{p}\right\}_{p=1 . .2 L}$ and right $\left\{v_{p}\right\}_{p=1 . . N}$ singular vectors of $H$ as columns. We assume that the $\lambda_{m}$ are indexed in increasing order. The condition number $c_{H}=\lambda_{N} / \lambda_{1} \geq 1$ is the ratio of the largest to the smallest singular value. For a poorly conditioned channel $c_{H}$ is large. The occurrence of poorly channels is high enough to cause a significant degradation of the average performance of the sub-optimal detection schemes [11]. In $\mathrm{ZF}$ equalization domain, we can write $X_{Z F}=$ $X+\tilde{B}$ where $\mathrm{X}$ is the undistorted data corrupted by correlated noise $\tilde{B}$ with covariance matrix $\operatorname{Cov}_{\tilde{B}}=\sigma^{2} V \Sigma^{-2} V^{*}$. The contour surfaces of the probability density function of $\tilde{B}$ are hyperellipsoids of which the $p^{t h}$ principal axis is given by the $p^{t h}$ singular vector $v_{p}$ with a length proportional to $\sigma / \lambda_{p}$. Thus, the optimal solution point $X_{s o l}$ of (3) can be far from the $X_{Z F}$ point if the line $\left(X_{s o l}, X_{Z F}\right)$ has 
roughly the same direction than one of the dominant axis noise. Thus, the idea is to select a subset $I$ of $\xi$ that contains the closest point to the first $D$ dominant axis noise. This selection is performed by a simple geometrical method, i.e., intersection of a line and an hyperplane. Then, the point of $I$ are evaluated and a local search is performed in the vicinity of the best one.

\subsection{The algorithm}

In the following, we give all steps of the HIS detection algorithm with parameters $D$ (the number of studied dominant axis noise) and $M$ (the number of selected candidates):

Input: the received vector $Y$, the channel matrix $H$.

Output: a nearly ML solution $X_{\text {sol }}$ of (3).

Pre - processing: Find the $D$ singular vectors $\left(v_{p}\right)_{p=1 . . D}$ associated to the smallest singular values of the channel matrix $H$. Compute the $D \times N$ values $\left(1 / v_{p}(i)\right)$ and compute $H^{-1}$. Note that this pre-processing step is performed only once for every new channel matrix $H$.

Step1: perform a ZF step: $X_{Z F}=H^{-1} \times Y$.

Step2: generate the $D$ references lines $\left\{\triangle_{1}, . ., \triangle_{D}\right\}$ defined by the point $X_{Z F}$ and vectors $\left\{v_{p}\right\}_{p=1 \ldots D}$.

$$
\triangle_{p}:\left\{Z \in \mathcal{R}^{N} / Z=X_{Z F}+\alpha v_{p}, \alpha \in \mathcal{R}\right\}
$$

Step3: create $D$ subset $\mathcal{I}_{p}=\operatorname{sign}\left(\mathcal{C} \cap \triangle_{p}\right)$ where $\mathcal{C}=$ $\left\{\cup_{i=1 . . N}^{s=-1,1} \mathcal{P}(i, s)\right)$ and $\mathcal{P}(i, s)$ is a hyperplane defined by $\left\{x \in \mathcal{R}^{N} / x(i)=s\right\}$. Let us study the intersection between a given line $\triangle_{p}$ and hyperplane $\mathcal{P}(i, s)$. Using (4), the problem is to find $\alpha_{p, i}^{s}$ i.e.:

$$
\alpha_{p, i}^{s} v_{p}(i)+X_{Z F}(i)=s
$$

The intersection between a line and an hyperplane generates two cases of study:

- If $v_{p}(i) \neq 0: \alpha_{p, i}^{s}=\frac{s-X_{Z F}(i)}{v_{p}(i)}$ then $\left.\left\{\mathcal{P}(i, s) \cap \triangle_{d}\right)\right\}=$ $\alpha_{p, i}^{s} v_{d}+X_{Z F}=L_{p, i}^{s}$. The returned point is $\tilde{L}_{p, i}^{s}$ the projection of $L_{p, i}^{s}$ on $\{-1,1\}^{N}$.

- If $v_{p}(i)=0$ then the returned point is simply the projection of $X_{Z F}$ on $\{-1,1\}^{N}$. Thus $\mathcal{I}_{p}=\cup_{i=1 . . N}^{s=-1,1} \tilde{L}_{p, i}^{s}$ contains at most $2 N$ points. Eventually, suppress the redundant points.

Step4: evaluate the euclidean distances between $Y$ and $H \times X$ for all $X \in \mathcal{I}_{p}$ where $p \in\{1, . ., D\}$.

Step5: from each subset $\mathcal{I}_{p}$, extract the $M$ points having the minimum euclidean distance to generate the set of $M \times D$ points $\mathcal{L}$. Eventually, suppress the redundant points. Step6: use each point $X$ of $\mathcal{L}$ as a starting point of a greedy search $\mathcal{G}$ of the minimum euclidean distance

Step7: select $X_{\text {sol }}$ as the point with minimum distance in $\mathcal{G}(\mathcal{L})$.

The principle of the greedy search is simple: starting from
$X$, all neighbour constellation points at an euclidean distance of 1 from $X$ (i.e. $N$ points) are evaluated. If $X$ has the smallest euclidean distance the process is stopped, other wise, the best point is selected and the process is re-iterated. Note that, since only one coordinate changes between two distance evaluations, important computation saving can be obtained (from $O\left(N^{2}\right)$ to $O(N)$ ). Let us give an example to clarify how the algorithm operates. The parameters used by the HIS algorithm are $N=4, D=1$ and $M=3$ :

$$
\begin{aligned}
H & =\left(\begin{array}{cccc}
-1.01 & -1.43 & 0.30 & -0.09 \\
-0.12 & -1.02 & 0.72 & -0.55 \\
-0.21 & -0.92 & 0.97 & -0.32 \\
0.68 & 1.17 & -0.34 & -0.72
\end{array}\right) \\
X_{e} & =\left[\begin{array}{llll}
1 & 1 & 1 & 1
\end{array}\right]^{T} \\
\nu & =\left[\begin{array}{llll}
0.18 & 0.50 & -0.67 & 0.53
\end{array}\right]^{T} \\
Y & =\left[\begin{array}{llll}
-2.05 & -0.48 & -1.16 & 1.33
\end{array}\right]^{T}
\end{aligned}
$$

The coordinates of $Y$ with respect to the lattice are $X_{Z F}=$ $H^{-1} Y=[2.83,-0.86,-1.37,0.06]^{T}$. The first dominant axis noise vector $v_{1}$ can be found using the SVD decomposition of matrix $H$. The algorithm generates a subset $\mathcal{I}_{1}$ of all intersection points between the line $\triangle_{1}$ defined as (4) and $\mathcal{C}$ (step 3). In this example, the generated subset is $\mathcal{I}_{1}=\left[X_{1}, X_{2}, X_{3}, X_{4}\right]$. For all points $X_{i} \in \mathcal{I}_{1}$, the algorithm calculates the cost function $\left\|Y-H X_{i}\right\|_{2}^{2}$ where $i \in\{1 . .4\}$ and sorts them in ascending order:

\begin{tabular}{||c|c|c||}
\hline Point & coordinates & $\left\|Y-H \times X_{i}\right\|_{2}^{2}$ \\
\hline$X_{1}$ & {$[1,1,1,-1]^{T}$} & 2.90 \\
\hline$X_{2}$ & {$[1,1,-1,-1]^{T}$} & 3.95 \\
\hline$X_{3}$ & {$[-1,1,1,-1]^{T}$} & 8.04 \\
\hline$X_{4}$ & {$[1,-1,-1,1]^{T}$} & 9.48 \\
\hline
\end{tabular}

Finally, step 7 gives:

\begin{tabular}{||c|c|c||}
\hline Point & coordinates & $\left\|Y-H \times \mathcal{G}\left(X_{i}\right)\right\|_{2}^{2}$ \\
\hline $\mathcal{G}\left(X_{1}\right)$ & {$[1,1,1,1]^{T}$} & 1.01 \\
\hline $\mathcal{G}\left(X_{2}\right)$ & {$[-1,1,-1,-1]^{T}$} & 2.43 \\
\hline $\mathcal{G}\left(X_{3}\right)$ & {$[-1,1,-1,-1]^{T}$} & 2.43 \\
\hline
\end{tabular}

The HIS generated solution is $X_{\text {sol }}=\mathcal{G}\left(X_{1}\right)=X_{e}$ which has the minimum euclidean distance. Note that $\mathcal{G}\left(X_{2}\right)=$ $\mathcal{G}\left(X_{3}\right)$

\section{COMPLEXITY AND PERFORMANCE OF "HIS"}

In this section, we give the complexity in terms of basic arithmetic operations required by the HIS algorithm and we evaluate its performance. 


\subsection{Computational complexity}

The HIS computation complexity depends on the parameters $D$ and $M$. Let $L=K$ and $N=2 K$ for simplicity. The algorithm can be subdivised in two parts. First a pre-processing, dependent only on a channel matrix $H$ and second a processing which depends on the received vector $Y$. The HIS algorithm has a low computational complexity $8 D M K^{3}$ real additions and $8 D M K^{2}$ real multiplications. Note that the SVD decomposition can be done in $4 D K^{2}$ if the method proposed in [12] is used. The HIS algorithm has two advantages comparing to the Sphere Decoding (SD). Firstly, the algorithm SD is lower bounded by an exponential function in $K$ [13]. This proves that the expected complexity of SD can not be polynomial. Secondly, the SD is a sequential algorithm but the proposed algorithm HIS overcomes this problem, since it is essentially parallel. In fact, the $D$ dominating axis noise can be processed in parallel, in each direction each point of the list $\mathcal{I}_{d}$ can be determined and evaluated in parallel. Once $\mathcal{L}$ is obtained, all greedy search function $\mathcal{G}$ can be performed in a parallel way. Note that, the $\mathcal{G}$ function is iterated only one to two times.

\subsection{Simulation results}

Finally, simulation results are given and the bit error rate performance of the proposed detection algorithm is presented with comparison to those of ZF, MMSE, PIC and SD algorithms. Fig. 1 shows the performance of HIS algorithm in a Rayleigh fading channel for a fully loaded downlink MC-CDMA system with $K=16$ users and employing an uncoded 4-QAM modulation. For a BER as low as $10^{-4}$, the HIS algorithm $D=M=4$ introduces only $0.2 \mathrm{~dB}$ degradation compared to the optimal Sphere Decoding algorithm againt $3.8 d B$ for the PIC (with two MMSE stages) algorithm.

\section{CONCLUSION}

In this paper, a new efficient algorithm named "HIS" is proposed which approximately solve the NP-hard ILS problem (3) with a polynomial-time complexity of $\mathcal{O}\left(K^{3}\right)$. Simulation results show that the HIS detector introduced only a small performance degradation compared to an ML detector. The inherent parallelism of the HIS algorithm allows high throughput and low complexity decoder. The new approach method can be efficiently employed in the case of Multiple Input Multiple Output (MIMO) system.

\section{ACKNOWLEDGMENT}

This research work has been supported by the PALMYRE CPER (https://palmyre.univ-ubs.fr/CPER/index.html) project

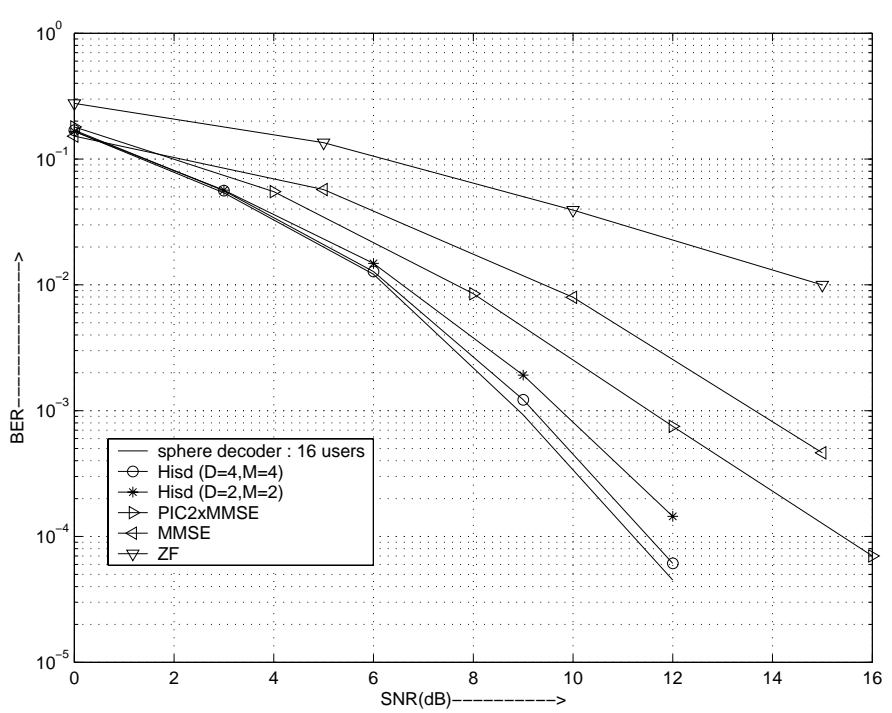

Fig. 1. Comparison between SD detection, HIS detection and others sub-optimum detector in a Rayleigh fading channel ( $K=16$ users)

founded by Brittany region, Morbihan Departement and Lorient Agglomeration Community. The authors like to thank Slim Chabbouh for his fruitful discussion on the current detector algorithms.

\section{REFERENCES}

[1] N. Yee, J. P. Linnartz, G. Fettweis, " Multi carrier CDMA Indoor Wireless Networks" in the proceedings of the IEEE PIMRC'93, pp.109 -113. Yokohama, Japan, 1993.

[2] S.Hara,R.Prasad," Overview of the Multi carrier CDMA " in the proceedings of the IEEE Communication Magazine, pp. 126 -133. Dec 1997.

[3] Sergio Verdù,"Multi-User Detection”. Ed. Cambridge University Press 1998. [4] S Moshavi, "Multi-User Detection for DS-CDMA Communication", IEEE Comm. Magazine, Oct 1996, p.124 - 136.

[5] K. Fazel and L. Papke, "On the performance of convolutionally coded CDMA OFDM for mobile communication system”, in Proc. PIMRC'93, Sept. 1993, pp. 468-472.

[6] L. Brunel, "Optimum multiuser detection for MC-CDMA systems using sphere decoding," in Proc. IEEE International Symposium on Personal, Indoor and Mobile Radio Communications, San Diego, California, Vol. 1, pp. A-16-A-20, Oct. 2001

[7] R. Lupas and S. Verdu, "Linear multiuser detector for synchronous code-division multiple-access channels," IEEE Trans. Info. Theory, IT-35:123-136, Jan. 1989.

[8] F. v.d. Wijk, G.J.M. Janssen, R. Prasad, "Groupwise successive interference cancellation in a DS-CDMA system", IEEE Proc. PIMRC'95, pp. 742 - 746, Toronto, Canada, September 1995.

[9] E. Viterbo and J. Boutros, "A universal lattice code decoder for fading channels," IEEE Trans. on Inf. Theory, pp. 1639-1642, July 1999.

[10] W.K. Ma, T.N. Davidson, K.M. Wong, Z.-Q. Luo, and P.C. Ching, "Quasi maximum likelihood multiuser detection using semi-definite relaxation with application to synchronous CDMA”, IEEE Trans. Sig. Proc., vol. 50, no. 4, pp. 912 - 922, April, 2002.

[11] H. Artes, D. Seethaler, F. Hlawatsch: "Efficient Detection Algorithms for MIMO Channels: A Geometrical Approach to Approximate ML Detection"; IEEE Transactions on Signal Processing, Vol. 51 (2003), 11; 2808 - 2820.

[12] G. H. Golub and C. F. Van Loan, Matrix Computations, 3rd ed. Baltimore: Johns Hopkins Univ. Press, 1996.

[13] J. Jaldn and B. Ottersten, "An exponential lower bound on the expected complexity of sphere decoding" in Proc.IEEE ICASSP'04, May 2004. 